\title{
Conewise Linear Systems: A Characterization of Transitive Cones in 3D-Space ${ }^{\star}$
}

\author{
A. B. Özgüler and M. Zakwan
}

\begin{abstract}
A spatial (3D) piecewise linear system with multiple modes having conewise state spaces is considered. A single mode in this system is called transitive from one (respectively, two) of its borders if every trajectory that starts in its interior or at a border travels in its interior, hits that border (respectively, one of the two borders), and goes out of the cone. This paper characterizes transitive cones in case the dynamics in the cone is dictated by real and distinct eigenvalues. An example of a 3D piecewise linear system composed of transitive cones illustrates how a nonlinear oscillator can be synthesized.
\end{abstract}

Keywords conewise linear systems, switched linear, oscillator, stability

\section{INTRODUCTION}

Piecewise linear systems find application in a range of engineering fields. Relays and saturations are some of the most common piecewise linear components found in control systems. Switches occur when a plant operates in different modes or under physical constraints. Diodes and transistors are naturally modeled as piecewise linear and are essential components in all electronic circuits. A global nonlinear model constructed from locally valid linearizations is easy to understand and would be readily accepted by any engineer, [11]. Any progress made, no matter how limited, on the stability problem of a PLS would help satisfy the most basic requirement in all these applications and play a key role at both analysis and design phases, [14], [16].

A conewise piecewise linear system (PLS) comprises multiple linear time invariant subsystems with states constrained to a cone and an autonomous switching among these subsystems, [3], [8], [2]. Although such nonlinear systems are obtained by an immediate extension of linear systems, their stability properties are surprisingly difficult to characterize. A complete characterization result for stability is limited to the very special two-state (planar, 2D) case, [10], [4], in the analysis of which a direct approach has been used as opposed to the more common Lyapunov methods. While Lyapunov approaches provide many sufficient conditions for stability of general (nD) PLS, the method becomes quickly stagnant by the requirement to concoct common Lyapunov functions for a set of subsystems [12], [11], [15], [13].

Finding conditions for stability of PLS is thus still an open problem and many books and survey papers are devoted to the subject [16], [14]. The point of view adopted here is a direct approach and focuses on the stability of 3D conewise

\footnotetext{
* This work is supported in full by the Science and Research Council of Turkey (TÜBİTAK) under project EEEAG-117E948.

The authors are with the Department of Electrical and Electronics Engineering, Bilkent University, 06800 Bilkent, Ankara, Turkey. Contact email: ozguler@ee.bilkent.edu.tr
}

PLS. It is similar in topic and method to those in [1], [6], [5]. The latter two papers focus on the stability of bimodal 3D PLS and gives a taste of the difficulties involved in coming up with necessary and sufficient conditions even under the assumption of certain special dynamics in the two modes.

Asymptotic stability of a conewise PLS is tightly dependent upon the transitivity properties of the individual modes. Ignoring well-posedness issues, we can consider an extreme situation. Suppose that all cones that make up the modes are "sinks," i.e. for all initial conditions starting in the cone all trajectories remain in the cone. Then, a necessary and sufficient condition for stability would be that all modes have Hurwitz $A$-matrices. What makes the stability (and the well-posedness) problem non-trivial is that some trajectories starting at the interior of a cone may hit a boundary at a finite time.

In our study, we focus on a question that is at the heart of the stability issue: when a single mode constrained in a 3D cone having three boundaries $k, l$, and $m$ (which are planar (2D) cones) is "transitive"? A mode is transitive from boundary $k$ if a trajectory starting in the interior or at any of the three boundaries goes out of the mode through boundary $k$. Similarly a mode may be transitive from two borders $l$ and $m$. In the case that dynamics in the mode are dictated by an A-matrix with real and distinct eigenvalues, we give a complete characterization of a $k$-transitive and $l m$-transitive conewise single mode. In doing this, we also identify those modes for which transitivity is a "structural" property, i.e. it is solely determined by the relative position of its eigenvectors to the boundary vectors that define the 3D cone. To illustrate the significance for stability of the property of transitivity, we show how to synthesize a 3D conewise PLS (that fills up the space) and examine its stability properties.

The paper is organized as follows. After general introduction of problem in Section I, Section II describes the characteristics of a single mode in PLS. In Section III, main results for transitivity properties of a single mode system are discussed along with an example. Finally, conclusion is drawn in Section IV. Although this paper contains no necessary and sufficient condition for stability, the example in Section III.B illustrates how the result of Theorem 1 and its corollary helps design a nonlinear oscillator by numerical experimentation.

Notation: We denote the real numbers, $n$-dimensional real vector space, and the set of real $n \times m$ matrices by $\mathbf{R}, \mathbf{R}^{n}$, and $\mathbf{R}^{n \times m}$, respectively. The norm of a vector $\mathbf{v} \in \mathbf{R}^{n}$ will be denoted by $|\mathbf{v}|$. If $\mathbf{v}, \mathbf{w} \in \mathbf{R}^{3}$, then $\mathbf{v} \times \mathbf{w}$ will 
denote the cross product of the vectors and $\mathbf{v} \cdot \mathbf{w}=\mathbf{v}^{T} \mathbf{w}$, their dot product, where ' $\mathrm{T}$ ' denotes 'transpose.' An arbitrary permutation of $(1,2,3)$ wlll be denoted by $(k, l, m)$.

\section{Conewise Piecewise Linear Systems}

The class of systems considered are

$$
\dot{\mathbf{x}}=\left\{\begin{array}{lll}
A_{1} \mathbf{x} & \text { if } & \mathbf{x} \in \mathbf{S}_{1}, \\
A_{2} \mathbf{x} & \text { if } & \mathbf{x} \in \mathbf{S}_{2}, \\
\vdots & \vdots & \vdots \\
A_{N} \mathbf{x} & \text { if } & \mathbf{x} \in \mathbf{S}_{N},
\end{array}\right.
$$

where $A_{i} \in \mathbf{R}^{3 \times 3}$ and, with $C_{i} \in \mathbf{R}^{3 \times 3}$,

$$
\mathbf{S}_{i}:=\left\{\mathbf{x} \in \mathbf{R}^{3}: C_{i} \mathbf{x} \geq 0\right\},
$$

for $i=1,2, \ldots, N$. We assume that each $C_{i}$ is nonsingular and is such that $\operatorname{det} C_{i}>0$. Note that the latter causes no loss of generality and only requires a permutation of rows of $C_{i}$ if necessary. The nonsingularity assumption implies that (1) is truly multi-modal $(N \geq 2)$ and that the interior of each $\mathbf{S}_{i}$, int $\mathbf{S}_{i}$, is nonempty. We further assume that the interior of each pairwise intersection int $\mathbf{S}_{i} \cap \mathbf{S}_{k}, i \neq k$ is empty and that $\mathbf{S}_{1} \cup \ldots \cup \mathbf{S}_{N}=\mathbf{R}^{3}$. These assumptions ensure, in the terminology of [10], that (1) is memoryless. Further, let

$$
S_{i}=\left[\begin{array}{lll}
\mathbf{s}_{i 1} & \mathbf{s}_{i 2} & \mathbf{s}_{i 3}
\end{array}\right]:=C_{i}^{-1}=\left[\begin{array}{c}
\mathbf{c}_{i 1}^{T} \\
\mathbf{c}_{i 2}^{T} \\
\mathbf{c}_{i 3}^{T}
\end{array}\right]^{-1}
$$

so that $\operatorname{det} S_{i}>0$. It is easy to see that, if each $\mathbf{S}_{i}, i=$ $1, \ldots, N$ is strictly contained in a half-space, then $\mathbf{S}_{i}$ is a convex cone $\mathbf{S}_{i}=$ cone $\left\{\mathbf{s}_{i 1}, \mathbf{s}_{i 2}, \mathbf{s}_{i 3}\right\}$, where cone $\left\{\mathbf{v}_{1}, \ldots, \mathbf{v}_{l}\right\}=$ $\left\{\alpha_{1} \mathbf{v}_{1}+\ldots+\alpha_{l} \mathbf{v}_{l}: \alpha_{j} \geq 0, j=1, \ldots, l\right\}$. The boundaries of each $\mathbf{S}_{i}$ are three planar cones

$$
\mathrm{B}_{i k}=\text { cone }\left\{\mathbf{s}_{i l}, \mathbf{s}_{i m}\right\}, k \neq l \neq m \in\{1,2,3\}
$$

Note that because det $S_{i}>0$, the cross products $\mathbf{s}_{i 1} \times \mathbf{s}_{i 2}$, $\mathbf{s}_{i 2} \times \mathbf{s}_{i 3}, \mathbf{s}_{i 3} \times \mathbf{s}_{i 1}$ are positively oriented according to the right-hand rule.

If, in (1), there is a mode defined on a half-space or a cone larger than a half-space, then it can be split into two modes having the same dynamics (the same A-matrix) so that each is still defined on a convex cone.

Given a mode $i$, its eigenvalues will be denoted by $\lambda_{i 1}, \lambda_{i 2}, \lambda_{i 3} \in \mathbf{C}$ and, in case of real eigenvalues, they will be indexed so that $\lambda_{i 1} \geq \lambda_{i 2} \geq \lambda_{i 3}$.

\section{A Single Mode}

We now focus on a single mode $i$ (and temporarily drop index $i$ that designates a mode) to consider

$$
\dot{\mathbf{x}}=A \mathbf{x}, \mathbf{x} \in \mathbf{S}=\text { cone }\left\{\mathbf{s}_{1}, \mathbf{s}_{2}, \mathbf{s}_{3}\right\},
$$

and $S=\left[\begin{array}{lll}\mathbf{s}_{1} & \mathbf{s}_{2} & \mathbf{s}_{3}\end{array}\right]$ with $\operatorname{det} S>0$. Let $\mathbf{v}_{1}, \mathbf{v}_{2}, \mathbf{v}_{3} \in \mathbf{R}^{3}$ be such that $A V=V \Lambda, V=\left[\begin{array}{lll}\mathbf{v}_{1} & \mathbf{v}_{2} & \mathbf{v}_{3}\end{array}\right]$, where $\Lambda$ is a Jordan form of $A$. Also let

$$
W=\left[\begin{array}{l}
\mathbf{w}_{1}^{T} \\
\mathbf{w}_{2}^{T} \\
\mathbf{w}_{3}^{T}
\end{array}\right]:=V^{-1} .
$$

Depending on whether the eigenvalues are real or non-real and distinct or repeated, there are a total of seven possible Jordan forms and $\mathbf{v}_{2}$ and/or $\mathbf{v}_{3}$ may be either generalized eigenvectors or the real or imaginary parts of non-real eigenvectors. The word "eigenvector" will refer to a true eigenvector. Let $\mathbf{x}(t, \mathbf{b})$ denote the solution (trajectory) of (2) resulting from an initial condition $\mathbf{b}$ inside the cone $\mathbf{S}$. Consider the identity $C V=(W S)^{-1}$ in explicit form

$$
\left[\begin{array}{lll}
\mathbf{c}_{1}^{T} \mathbf{v}_{1} & \mathbf{c}_{1}^{T} \mathbf{v}_{2} & \mathbf{c}_{1}^{T} \mathbf{v}_{3} \\
\mathbf{c}_{2}^{T} \mathbf{v}_{1} & \mathbf{c}_{2}^{T} \mathbf{v}_{2} & \mathbf{c}_{2}^{T} \mathbf{v}_{3} \\
\mathbf{c}_{3}^{T} \mathbf{v}_{1} & \mathbf{c}_{3}^{T} \mathbf{v}_{2} & \mathbf{c}_{3}^{T} \mathbf{v}_{3}
\end{array}\right]\left[\begin{array}{lll}
\mathbf{w}_{1}^{T} \mathbf{s}_{1} & \mathbf{w}_{1}^{T} \mathbf{s}_{2} & \mathbf{w}_{1}^{T} \mathbf{s}_{3} \\
\mathbf{w}_{2}^{T} \mathbf{s}_{1} & \mathbf{w}_{2}^{T} \mathbf{s}_{2} & \mathbf{w}_{2}^{T} \mathbf{s}_{3} \\
\mathbf{w}_{3}^{T} \mathbf{s}_{1} & \mathbf{w}_{3}^{T} \mathbf{s}_{2} & \mathbf{w}_{3}^{T} \mathbf{s}_{3}
\end{array}\right]=I
$$

Let $(k, l, m)$ be any permutation of $(1,2,3)$. Then, $\mathbf{c}_{k}^{T} \mathbf{v}_{i}=0$ if and only if $\mathbf{v}_{i}$ is in $\mathbf{s}_{m} \mathbf{s}_{l}$-plane. Similarly, $\mathbf{w}_{m}^{T} \mathbf{s}_{i}=0$ if and only if $\mathbf{s}_{i}$ is in $\mathbf{v}_{m} \mathbf{v}_{l}$-plane, for any $i \in\{1,2,3\}$.

We will refer to a single mode as well-posed if $\mathbf{b} \in \mathrm{B}_{k}$, then for some $\epsilon>0$ and for all $t \in(0, \epsilon], \mathbf{x}(t, \mathbf{b}) \notin \mathrm{B}_{k}$, $k=1,2,3$, i.e., every trajectory starting at a boundary either goes out of $\mathbf{S}$ or goes into int $\mathbf{S}$ (no matter for how small a time). If there is a real eigenvector at a boundary, then any trajectory that starts in the direction of that eigenvector will remain in that direction so that well-posedness is clearly violated.

Definition 1. A nonzero vector $\mathbf{v}$ is exterior to $\mathbf{S}$ if for all $\alpha \in \mathbf{R}$ it holds that $\alpha \mathbf{v} \notin \mathbf{S}$. It is interior to $\mathbf{S}$ if for some $\alpha \neq 0, \alpha \mathbf{v} \in \mathbf{S}$.

Fact 1. A real eigenvector $\mathbf{v}_{k}$ of $A$ is exterior to $\mathbf{S}$ if and only if all entries of the $k$-th column of the matrix $C V$ are nonzero and do not have the same sign, i.e. iff the set $\left\{\mathbf{c}_{1}^{T} \mathbf{v}_{k}, \mathbf{c}_{2}^{T} \mathbf{v}_{k}, \mathbf{c}_{3}^{T} \mathbf{v}_{k}\right\}$ has a positive and a negative element.

Proof. By definition, a vector $\mathbf{v}$ is interior to $\mathbf{S}$ if and only if $\left\{\mathbf{c}_{h}^{T} \mathbf{v}=\left(\mathbf{s}_{i} \times \mathbf{s}_{j}\right) \cdot \mathbf{v}\right.$ have the same sign for any positive permutation $(i, j, h)$ of $(1,2,3)$. Hence, this is true for $\mathbf{v}_{k}$ if and only if all entries of the column $k$ of $C V$ have the same sign. It is exterior to $\mathbf{S}$ if and only if it is not interior to $\mathbf{S}$.

Definition 2. i) A mode is $k$-transitive for $k \in\{1,2,3\}$ if for every $0 \neq \mathbf{b} \in \mathbf{S}$, there exists a finite $t_{k}^{*}>0$ such that $\mathbf{x}\left(t_{k}^{*}, \mathbf{b}\right) \in \mathrm{B}_{k}$ and $\mathbf{x}(t, \mathbf{b}) \notin \mathrm{B}_{l} \cup \mathrm{B}_{m}$ for any $t \in\left(0, t_{k}^{*}\right)$. ii) A mode is $l m$-transitive for $l, m \in\{1,2,3\}$ if for every $0 \neq \mathbf{b} \in \mathbf{S}$, there exists a finite $t_{k}^{*}>0$ such that $\mathbf{x}\left(t_{k}^{*}, \mathbf{b}\right) \in$ $\mathrm{B}_{l} \cup \mathrm{B}_{m}$ and $\mathbf{x}(t, \mathbf{b}) \notin \mathrm{B}_{k}$ for any $t \in\left(0, t_{k}^{*}\right)$.

In a $k$-transitive mode, all trajectories starting anywhere in $\mathbf{S}$, including all three boundaries, hit the boundary- $k$ before hitting any of the other two and exit the cone from that boundary. In an $l m$-transitive mode, on the other hand, trajectories starting in $\mathbf{S}$, including boundaries $l$ and $m$, avoid the boundary- $k$ until they hit one of the other two; a trajectory starting at boundary $k$ goes into the cone and exits again from one of the other two.

We now give expressions for trajectories $\mathbf{x}(t, \mathbf{b})$ of (2) resulting from an initial condition $\mathbf{b} \in \mathbf{S}$, determine the conditions under which trajectories may intersect one of the boundaries $\mathrm{B}_{k}=$ cone $\left\{\mathbf{s}_{l}, \mathbf{s}_{m}\right\}$, and derive the expressions for the values of such trajectories at the boundaries of $\mathbf{S}$. We will assume that the single mode is well-posed. 


\section{A. Real and Distinct Eigenvalues $\lambda_{1}>\lambda_{2}>\lambda_{3}$}

The Jordan form in this case is $\Lambda=\operatorname{diag}\left\{\lambda_{1}, \lambda_{2}, \lambda_{3}\right\}$ and all eigenvectors are real. By well-posedness assumption, we have that none is at a boundary, i.e.,

$$
\mathbf{c}_{k}^{T} \mathbf{v}_{i} \neq 0, \quad k, i=1,2,3,
$$

The unique solution of (2) for the initial state at $\mathbf{b}$, the trajectory, is given by

$$
\mathbf{x}(t, \mathbf{b})=e^{\lambda_{1} t} \mathbf{w}_{1}^{T} \mathbf{b} \mathbf{v}_{1}+e^{\lambda_{2} t} \mathbf{w}_{2}^{T} \mathbf{b} \mathbf{v}_{2}+e^{\lambda_{3} t} \mathbf{w}_{3}^{T} \mathbf{b} \mathbf{v}_{3} .
$$

This hits the $\mathbf{s}_{l} \mathbf{s}_{m}$-plane at a finite time $t_{k}>0$ if and only if $\mathbf{c}_{k}^{T} \mathbf{x}\left(t_{k}, \mathbf{b}\right)=0$, where

$$
\mathbf{c}_{k}^{T} \mathbf{x}(t, \mathbf{b})=e^{\lambda_{1} t} n_{k 1}+e^{\lambda_{2} t} n_{k 2}+e^{\lambda_{3} t} n_{k 3},
$$

with $n_{k i}:=\mathbf{c}_{k}^{T} \mathbf{v}_{i} \mathbf{w}_{i}^{T} \mathbf{b}, i=1,2,3$.

If $\mathbf{b} \in \mathbf{R}^{n}$, then the trajectory $\mathbf{x}\left(t_{k}, \mathbf{b}\right)=0$ hits the border $\mathrm{B}_{k}$ at time $t_{k}>0$ and is moving towards $\mathbf{s}_{k}$ if and only if $\mathbf{c}_{k}^{T} \mathbf{x}\left(t_{k}, \mathbf{b}\right)=0$ and $\mathbf{c}_{k}^{T} \dot{\mathbf{x}}\left(t_{k}, \mathbf{b}\right)>0$. It moves towards $-\mathbf{s}_{k}$ if and only if $\mathbf{c}_{k}^{T} \dot{\mathbf{x}}\left(t_{k}, \mathbf{b}\right)<0$. We can thus write

$$
\begin{aligned}
& \mathbf{c}_{k}^{T} \dot{\mathbf{x}}\left(t_{k}, \mathbf{b}\right)=\left(\lambda_{1}-\lambda_{3}\right) e^{\lambda_{2} t_{k}}\left[e^{\left(\lambda_{1}-\lambda_{2}\right) t_{k}} n_{k 1}+p n_{k 2}\right] \\
& \quad=\left(\lambda_{1}-\lambda_{3}\right) e^{\lambda_{3} t_{k}}\left[e^{\left(\lambda_{1}-\lambda_{3}\right) t_{k}}(1-p) n_{k 1}-p n_{k 3}\right] \\
& \quad=\left(\lambda_{1}-\lambda_{3}\right) e^{\lambda_{3} t_{k}}\left[-e^{\left(\lambda_{2}-\lambda_{3}\right) t_{k}}(1-p) n_{k 2}-n_{k 3}\right] .
\end{aligned}
$$

where $p:=\frac{\lambda_{2}-\lambda_{3}}{\lambda_{1}-\lambda_{3}}$, and check the sign in order to determine the direction of a trajectory after hitting the border. (This analysis yields the last column of Table 1 below.)

If on the other hand, $\mathbf{b} \in \mathrm{B}_{k}$, then $\mathbf{c}_{k}^{T} \mathbf{b}=0$ and $n_{k 1}+$ $n_{k 2}+n_{k 3}=0$ so that

$$
n_{k 1}+p n_{k 2}=(1-p) n_{k 1}-p n_{k 3}=-(1-p) n_{k 2}-n_{k 3} .
$$

Fact 2. A single mode is not well-posed if and only if for some $k \in\{1,2,3\}$, there exists $\mathbf{b} \in \mathrm{B}_{k}$ for which (5) is zero.

Proof. If $\mathbf{b} \in \mathrm{B}_{k}$, then $\mathbf{c}_{k}^{T} \mathbf{b}=0$ so that equality (5) holds. The trajectory starting at such $\mathbf{a} \mathbf{b}$ remains in $\mathrm{B}_{k}$ if and only if $\mathbf{c}_{k}^{T} \dot{\mathbf{x}}(0, \mathbf{b})=0$, which holds if and only if (5) is zero.

We caution here that the mode being well-posed discards the possibility of a trajectory being tangent to $\mathrm{B}_{k}$ at any $t \geq$ 0 . This is, of course a major assumption and eliminates the necessity of dealing with the second and higher derivatives of $\mathbf{c}_{k}^{T} \mathbf{x}(t, \mathbf{b})$. Also note that if (5) is positive (resp., negative), then the trajectory starting at $\mathrm{B}_{k}$ moves in the direction of $\mathbf{s}_{k}$ (resp., $-\mathbf{s}_{k}$ ), and conversely. (The question of "under exactly what conditions on the properties of $A$ matrices (5) is zero" is avoided here. We only note here that it is closely tied to the conditions given in [9] for the bimodal case.)

\begin{tabular}{|c|c|c|c|c|c|c|}
\hline Type & $n_{k 1}$ & $n_{k 2}$ & $n_{k 3}$ & Exists & $\# t_{k}$ & Moves \\
\hline \hline 1 & - & - & + & yes & 1 & out \\
\hline 2 & - & + & + & yes & 1 & out \\
\hline 3 & - & + & - & yes & 1 & C(b) \\
\hline 4 & + & - & + & C (a) & 2 & C(c) \\
\hline 5 & + & - & - & no & 0 & - \\
\hline 6 & + & + & - & no & 0 & - \\
\hline 7 & + & + & + & no & 0 & - \\
\hline 8 & - & - & - & no & 0 & - \\
\hline
\end{tabular}

TABLE I: Conditions on $\mathbf{b} \in$ int $\mathbf{S}$ for exit from $\mathrm{B}_{k}$

$$
\begin{aligned}
& \mathrm{C}(\mathrm{a}) \text { : yes } \Leftrightarrow\left(\frac{-p n_{k 2}}{n_{k 1}}\right)^{\frac{p}{1-p}} \geq \frac{-n_{k 3}}{(1-p) n_{k 2}}, \frac{-p n_{k 2}}{n_{k 1}}>1 \\
& \mathrm{C}(\mathrm{b}): \text { out } \Leftrightarrow \frac{-p n_{k 2}}{n_{k 1}}<e^{\left(\lambda_{1}-\lambda_{2}\right) t_{k}} \\
& \mathrm{C}(\mathrm{c}) \text { : out } \Leftrightarrow \frac{-p n_{k 2}}{n_{k 1}}>e^{\left(\lambda_{1}-\lambda_{2}\right) t_{k}}
\end{aligned}
$$

Lemma 1. Let $(k, l, m)$ be a positive permutation of $(1,2,3)$ and let $\mathbf{S}=$ cone $\left\{\mathbf{s}_{1}, \mathbf{s}_{2}, \mathbf{s}_{3}\right\}$ be positively oriented. A trajectory (4), starting with $\mathbf{b} \in$ int $\mathbf{S}$, intersects $\mathbf{s}_{l} \mathbf{S}_{m^{-}}$ plane at some $t=t_{k}>0$ and goes out of $\mathbf{S}$ if and only at least two among $\left\{n_{k 1}, n_{k 2}, n_{k 3}\right\}$ are nonzero and one of the following (i)-(iv) holds:

i) $\quad n_{k 1}<0, n_{k 3}>0$,

ii) $\quad n_{k 1}<0, n_{k 3}<0, n_{k 2}>0$, and at the time of intersection $C(b)$ holds,

iii) $\quad n_{k 1}>0, n_{k 3}>0, n_{k 2}<0, C(a)$ holds, and at a time of intersection $C(c)$ holds,

iv)

$$
\begin{aligned}
& n_{k 1}=0, n_{k 3}>-n_{k 2}>0, \text { or } \\
& n_{k 2}=0, n_{k 3}>-n_{k 1}>0, \text { or } \\
& n_{k 3}=0, n_{k 2}>-n_{k 1}>0 .
\end{aligned}
$$

Table 1 summarizes the results of Lemma 1 by excluding singular cases of $\mathbf{w}_{1}^{T} \mathbf{b}=0, \mathbf{w}_{2}^{T} \mathbf{b}=0$, or $\mathbf{w}_{3}^{T} \mathbf{b}=0$ (initial condition being on one of the $\mathbf{v}_{k} \mathbf{v}_{l}$-planes). A sketch of the proof of Lemma 1 is given in the Appendix.

Definition 3. A cone $\left\{\mathbf{s}_{1}, \mathbf{s}_{2}, \mathbf{s}_{3}\right\}$ excludes cone $\left\{\mathbf{v}_{1}, \mathbf{v}_{2}, \mathbf{v}_{3}\right\}$ if $\left\{\mathbf{v}_{1}, \mathbf{v}_{2}, \mathbf{v}_{3}\right\}$ are exterior to it and neither of the three planes cone $\left\{\mathbf{v}_{1}, \mathbf{v}_{2},\right\}$, cone $\left\{\mathbf{v}_{2}, \mathbf{v}_{3}\right\}$, cone $\left\{\mathbf{v}_{3}, \mathbf{v}_{1}\right\}$ has an intersection with it. Two cones are mutually exclusive if both exclude one another.

It is straightforward to see that cone $\left\{\mathbf{s}_{1}, \mathbf{s}_{2}, \mathbf{s}_{3}\right\}$ excludes cone $\left\{\mathbf{v}_{1}, \mathbf{v}_{2}, \mathbf{v}_{3}\right\}$ if and only if each row of the matrix $W S$ has constant sign and each column of $C V$ has mixed signs. They are mutually exclusive if and only if both $W S$ and $C V$ have constant row signs with mixed signs in every column. The notation $\operatorname{rs}\{C V\}=(-,-,+)$, for instance, will mean that the first and second rows of $C V$ contain negative, and the third, positive entries. In Fig. 1, sign patterns for $C V$ and $W S$ corresponding to two possible mutually exclusive cases are given together with a bird's view of the cones cone $\left\{\mathbf{s}_{1}, \mathbf{s}_{2}, \mathbf{s}_{3}\right\}$, cone $\left\{\mathbf{v}_{1}, \mathbf{v}_{2}, \mathbf{v}_{3}\right\}$ of boundary vectors and the eigenvectors. The origin is down inside the page and the large and small triangles are cross sections of the two cones. This illustration assumes positively oriented triplet of vectors.

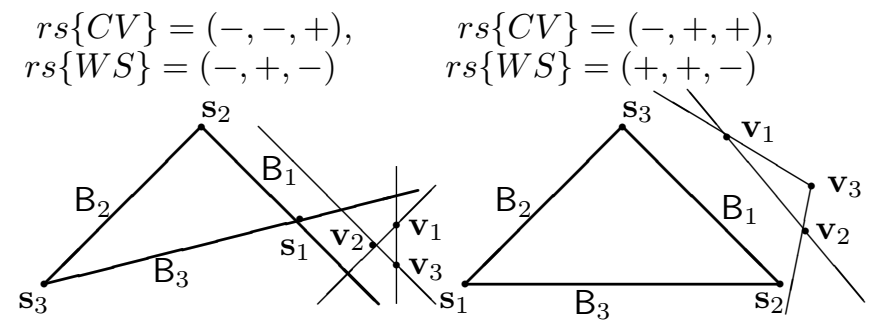

Fig. 1: Two mutually exclusive $\mathbf{S}$ and cone $\left\{\mathbf{v}_{1}, \mathbf{v}_{2}, \mathbf{v}_{3}\right\}$ cases with sign patterns of $C V, W S$ matrices 
A consequence of $W S$ having constant row signs is that signs of the entries of the column vector $W \mathbf{b}=$ $\left[\mathbf{w}_{1}^{T} \mathbf{b} \mathbf{w}_{2}^{T} \mathbf{b} \mathbf{w}_{3}^{T} \mathbf{b}\right]^{T}$ are constant over $\mathbf{b} \in \mathbf{S}$. If, in addition, $C V$ has also constant row sign, then it follows that signs of the triplets $\left(n_{i 1}, n_{i 2}, n_{i 3}\right)$ are also constant over $\mathbf{b} \in \mathbf{S}$. Having mixed column signs in $W S$ and $C V$ implies that every triplet has mixed signs and that one triplet for $i=$ $k, l, m$ has the negative sign pattern of the other two. In Fig. 1 , for instance, the sign patterns are, respectively,

\begin{tabular}{c|ccc} 
& $n_{i 1}$ & $n_{i 2}$ & $n_{i 3}$ \\
\hline$i=1$ & + & - & + \\
$i=2$ & + & - & + \\
$i=3$ & - & + & -
\end{tabular}

\begin{tabular}{c|ccc} 
& $n_{i 1}$ & $n_{i 2}$ & $n_{i 3}$ \\
\hline$i=1$ & - & - & + \\
$i=2$ & + & + & - \\
$i=3$ & + & + & -
\end{tabular}

Theorem 1. Let $(k, l, m)$ be a positive permutation of $(1,2,3)$ and let $\mathbf{S}=$ cone $\left\{\mathbf{s}_{1}, \mathbf{s}_{2}, \mathbf{s}_{3}\right\}$ be positively oriented. Let a mode $\mathbf{S}$ with real eigenvalues $\lambda_{1}>\lambda_{2}>\lambda_{3}$ be mutually exclusive with its cone of eigenvectors cone $\left\{\mathbf{v}_{1}, \mathbf{v}_{2}, \mathbf{v}_{3}\right\}$. $a$. The cone $\mathbf{S}$ is $k$-transitive if and only if it holds that
i) $\quad(1-p) n_{k 1}-p n_{k 3}<0$
$\forall \mathbf{b} \in \mathrm{B}_{k}$
ii) $\quad(1-p) n_{l 1}-p n_{l 3}>0$
$\forall \mathbf{b} \in \mathrm{B}_{l}$,
iii) $(1-p) n_{m 1}-p n_{m 3}>0$
$\forall \mathbf{b} \in \mathrm{B}_{m}$.

$b$. The cone $\mathbf{S}$ is lm-transitive if and only if it holds that

$$
\begin{array}{lll}
i v) & (1-p) n_{k 1}-p n_{k 3}>0 & \forall \mathbf{b} \in \mathrm{B}_{k}, \\
\text { v) } & (1-p) n_{l 1}-p n_{l 3}<0 & \forall \mathbf{b} \in \mathrm{B}_{l}, \\
\text { vi) } & (1-p) n_{m 1}-p n_{m 3}<0 & \forall \mathbf{b} \in \mathrm{B}_{m} .
\end{array}
$$

Proof. We first note that any trajectory that starts in the cone must eventually go out of the cone since all eigenvectors are exterior. It must thus intersect one of the border planes at a nonzero point (as the origin is a global equilibrium point). By the fact that $\mathbf{S}$ and cone $\left\{\mathbf{v}_{1}, \mathbf{v}_{2}, \mathbf{v}_{3}\right\}$ are mutually exclusive, both $W S$ and $C V$ have constant row signs with mixed signs in every column. This implies that each $n_{i j}, i, j=1,2,3$ has constant sign over $\mathbf{b} \in \mathbf{S}$ and that every triplet $\left(n_{i 1}, n_{i 2}, n_{i 3}\right)$ has mixed signs with one triplet for $i=k, l, m$ having negative sign pattern of the other two. This, in particular, eliminates the possibilities of Type- 7 and Type 8 of Table 1 in our mutually exclusive case.

The necessity of the conditions (i)-(iii) for $k$-transitivity and, of (iv)-(vi) for $l m$-transitivity, is clear since the required transitivity properties need to be satisfied by initial conditions starting at a border as well.

If (i) holds, then $n_{k 1}>0, n_{k 3}<0$ is not possible on $\mathrm{B}_{k}$ and hence anywhere in $\mathbf{S}$, which implies that $\left(n_{k 1}, n_{k 2}, n_{k 3}\right)$ is of Type- 1 to Type- 4 of Table 1. The conditions (ii) and (iii) eliminate the possibility of Type 1 and Type 2 for both borders $\mathrm{B}_{l}$ and $\mathrm{B}_{m}$. This means that if $\left(n_{k 1}, n_{k 2}, n_{k 3}\right)$ is of Type-1 or Type- 2 , then $\left(n_{l 1}, n_{l 2}, n_{l 3}\right)$ and $\left(n_{m 1}, n_{m 2}, n_{m 3}\right)$ are of Type- 5 or Type- 6 so that $\mathrm{B}_{k}$ is the only possible border of exit and the mode is $k$-transitive. If $\left(n_{k 1}, n_{k 2}, n_{k 3}\right)$ is of Type- 3 or Type- 4 , then all three $\left(n_{i 1}, n_{i 2}, n_{i 3}\right)$ are of Type- 3 or Type- 4 of Table 1 . The conditions on direction of trajectories imposed on the border planes by (i)-(iii) ensure that trajectories are all exiting via $\mathrm{B}_{k}$. This establishes that (i)-(iii) are sufficient for $k$-transitivity.
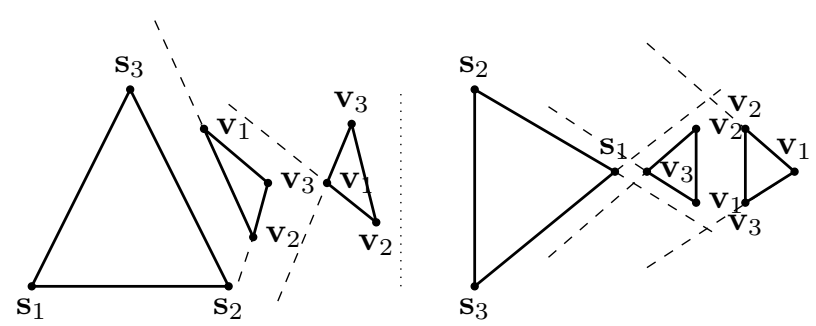

Fig. 2: Positions of the cone $\left\{\mathbf{v}_{1}, \mathbf{v}_{2}, \mathbf{v}_{3}\right\}$ relative to cone $\left\{\mathbf{s}_{1}, \mathbf{s}_{2}, \mathbf{s}_{3}\right\}$ for $k$-transitive

If (vi) holds, then $\left(n_{k 1}, n_{k 2}, n_{k 3}\right)$ is not of Type- 1 or Type2 of Table 1, whereas, by (iv) and (v), $\left(n_{l 1}, n_{l 2}, n_{l 3}\right)$ and $\left(n_{m 1}, n_{m 2}, n_{m 3}\right)$ are not of Type-5 or Type-6. It follows that $\left(n_{k 1}, n_{k 2}, n_{k 3}\right)$ is of Type-5 or Type-6, in which case the mode is $l m$-transitive, or it is of Type- 3 or Type 4 , in which case all borders are of Type- 3 or Type 4 . If (b.ii) holds, then all three $\left(n_{i 1}, n_{i 2}, n_{i 3}\right)$ are of Type- 3 or Type- 4 of Table 1. The conditions on direction of trajectories imposed on the border planes ensure that trajectories are all exiting via $B_{l}$ or $\mathrm{B}_{m}$. This establishes that (iv)-(vi) are sufficient for $l m$ transitivity.

Remark. Since conditions (i)-(vi) of Theorem 1 need be satisfied at the respective borders, they can be replaced by similar conditions of (5) by replacing the pair $\left(n_{i 1}, n_{i 3}\right)$ by $\left(n_{i 1}, n_{i 2}\right)$ or by $\left(n_{i 2}, n_{i 3}\right)$.

Let us call a property of a mode structural if it only depends on the eigenvalues and the eigenvectors of that mode. It will be noticed that the conditions on Theorem 1.a and 1.b may sometimes be determined solely with the signs of $\left\{n_{k 1}, n_{k 2}, n_{k 3}\right\}$, which are in turn constant over the whole cone in the mutually exclusive case. This observation gives the following structural properties.

Corollary 1. Suppose the hypotheses of Theorem 1 hold. Then,

a. The cone $\mathbf{S}$ is structurally $k$-transitive if and only if it holds that

$\begin{array}{lll}\text { i) } & n_{k 1}<0, n_{k 3}>0 & \text { for } \mathbf{b}=\mathbf{s}_{l} \text { and } \mathbf{b}=\mathbf{s}_{m}, \\ \text { ii) } & n_{l 1}>0, n_{l 3}<0 & \text { for } \mathbf{b}=\mathbf{s}_{m} \text { and } \mathbf{b}=\mathbf{s}_{k}, \\ \text { iii) } & n_{m 1}>0, n_{m 3}<0 & \text { for } \mathbf{b}=\mathbf{s}_{k} \text { and } \mathbf{b}=\mathbf{s}_{m} .\end{array}$

$b$. The cone $\mathbf{S}$ is structurally lm-transitive if and only if it holds that

$$
\begin{array}{lll}
\text { iv) } & n_{k 1}>0, n_{k 3}<0 & \text { for } \mathbf{b}=\mathbf{s}_{l} \text { and } \mathbf{b}=\mathbf{s}_{m} \\
\text { v) } & n_{l 1}<0, n_{l 3}>0 & \text { for } \mathbf{b}=\mathbf{s}_{m} \text { and } \mathbf{b}=\mathbf{s}_{k} \\
\text { vi) } & n_{m 1}<0, n_{m 3}>0 & \text { for } \mathbf{b}=\mathbf{s}_{k} \text { and } \mathbf{b}=\mathbf{s}_{m}
\end{array}
$$

The result stated in Corollary 1 depends only on the relative position of the triple of vectors $\left\{\mathbf{s}_{1}, \mathbf{s}_{2}, \mathbf{s}_{3}\right\}$ and $\left\{\mathbf{v}_{1}, \mathbf{v}_{2}, \mathbf{v}_{3}\right\}$. For instance, suppose that these mutually exclusive triplets are both positively oriented in space. Then all possible geometric configurations are illustrated in Fig. 2 and Fig. 3.

\section{B. An Example of a Spatial PLS with All Transitive Modes}

We now give an example that illustrates how a spatial PLS can be synthesized out of transitive modes that all obey the conditions of Corollary 1 (or of Theorem 1). One possible 

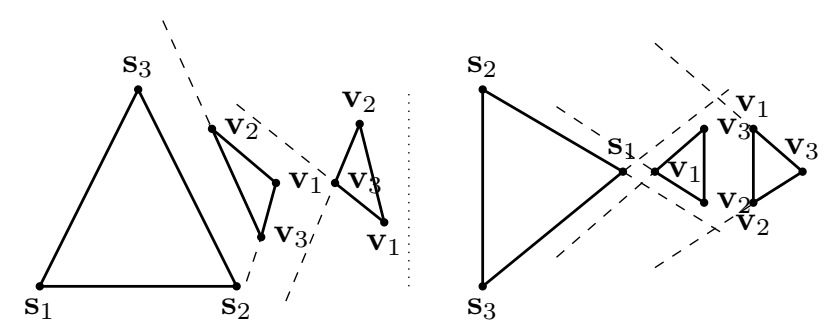

Fig. 3: Positions of the cone $\left\{\mathbf{v}_{1}, \mathbf{v}_{2}, \mathbf{v}_{3}\right\}$ relative to cone $\left\{\mathbf{s}_{1}, \mathbf{s}_{2}, \mathbf{s}_{3}\right\}$ for $l m$-transitive

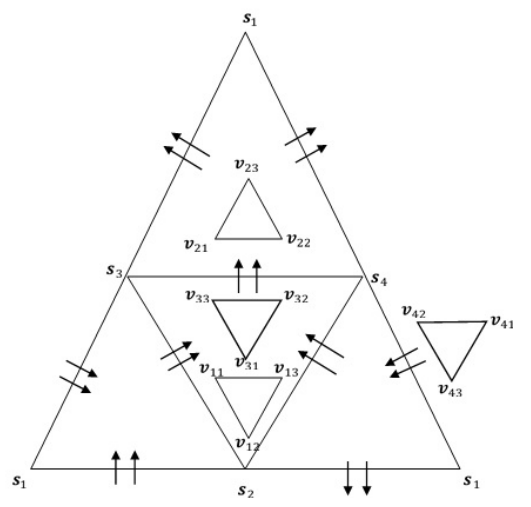

Fig. 4: A four-mode PLS with structurally transitive modes

application of the example in this section is in the design of a PLS oscillator that can be realized as an RC-circuit having piecewise-linear resistors.

The minimal number of the convex cones to fill-up $\mathbf{R}^{3}$ is known to be four, Ch. 2 of [7]. Consider four modes

$$
\dot{\mathbf{x}}=\left\{\begin{array}{lll}
A_{1} \mathbf{x} & \text { if } & \mathbf{x} \in \mathbf{S}_{1}, \\
A_{2} \mathbf{x} & \text { if } & \mathbf{x} \in \mathbf{S}_{2}, \\
A_{3} \mathbf{x} & \text { if } & \mathbf{x} \in \mathbf{S}_{3}, \\
A_{4} \mathbf{x} & \text { if } & \mathbf{x} \in \mathbf{S}_{4}
\end{array}\right.
$$

for which the cones are defined by $\mathbf{S}_{1}=$ cone $\left\{\mathbf{s}_{1}, \mathbf{s}_{2}, \mathbf{s}_{3}\right\}, \mathbf{S}_{2}=$ cone $\left\{\mathbf{s}_{2}, \mathbf{s}_{4}, \mathbf{s}_{3}\right\}$,

$\mathbf{S}_{3}=$ cone $\left\{\mathbf{s}_{4}, \mathbf{s}_{1}, \mathbf{s}_{3}\right\}, \mathbf{S}_{4}=$ cone $\left\{\mathbf{s}_{1}, \mathbf{s}_{4}, \mathbf{s}_{2}\right\}$, where the boundary vectors are $\mathbf{s}_{1}=\left[\begin{array}{ccc}1 & 0 & 0\end{array}\right]^{T}, \mathbf{s}_{2}=$ $\left[\begin{array}{lll}0 & 1 & 0\end{array}\right]^{T}, \mathbf{s}_{3}=\left[\begin{array}{lll}0 & 0 & 1\end{array}\right]^{T}, \mathbf{s}_{4}=\left[\begin{array}{lll}-1 & -1 & -1\end{array}\right]^{T}$. All four modes shown in the Fig. 4 are chosen to be structurally transitive using the conditions of Corollary 1. Note, for instance that the first mode is transitive from the plane cone $\left\{\mathbf{s}_{2}, \mathbf{s}_{2}\right\}$ with eigenvectors (positioned inside the second cone) in such a way that they are mutually exclusive and are posititoned according to the leftmost configuration in Fig.2. In order to obtain a PLS that is stable, unstable, and purely oscillatory, we have numerically manipulated the magnitude and the sign of the eigenvalues that are common to all four modes. The stability properties are verified numerically by sweeping over as many initial conditions as practicable in the four cones.

The matrix of eigenvectors $V_{i}$ in the expressions $A_{i}=$ $V_{i} \Lambda_{i} W_{i}$ in (6) are chosen as

$$
V_{1}=\left[\begin{array}{ccc}
-2 & -1 & -1 \\
1 & 7 & 1 \\
5 & 1 & 2
\end{array}\right], V_{2}=\left[\begin{array}{ccc}
-1 & -7 & -1 \\
-3 & -8 & -2 \\
4 & -6 & 1
\end{array}\right]
$$

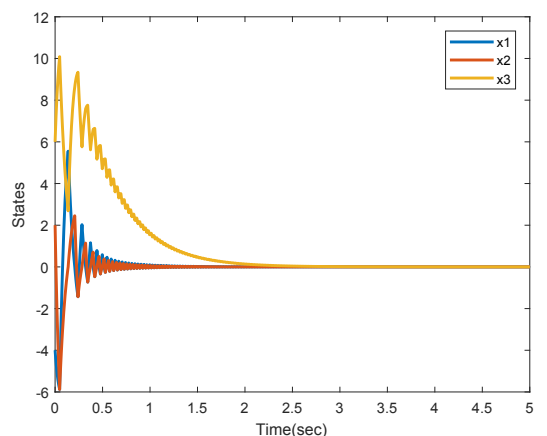

Fig. 5: State trajectories in stable case

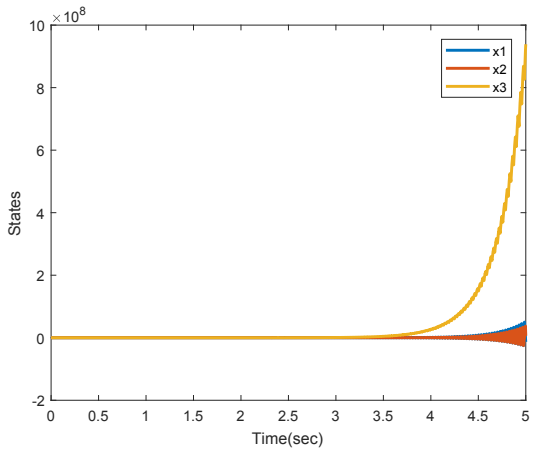

Fig. 6: State trajectories in unstable case

$$
V_{3}=\left[\begin{array}{ccc}
-11.5 & -9.5 & -6 \\
-4.5 & -5 & -1 \\
0 & -4 & 7
\end{array}\right], V_{4}=\left[\begin{array}{ccc}
1 & -0.5 & 7.5 \\
-5 & -5.5 & -4 \\
-2 & -2.5 & -1.5
\end{array}\right]
$$

and it is straightforward to verify that these choices make all four modes structurally transitive with transitivity properties as illustrated in Fig. 4.

For simplicity and for ease of adjustment of magnitudes that result in a stable, unstable, or (pure) oscillatory (6), the eigenvalues are chosen the same for all four modes in each case. Thus, for $i=1$ to $4,\left(\lambda_{i 1}=-7, \lambda_{i 2}=-8, \lambda_{i 3}=-9\right)$ yields a stable, $\left(\lambda_{i 1}=-1, \lambda_{i 2}=-2, \lambda_{i 3}=-3\right)$ yields an unstable , and $\left(\lambda_{i 1}=-4.538, \lambda_{i 2}=-5.3, \lambda_{i 3}=-6.6\right)$ yields an oscillatory PLS. The designs are validated by sweeping over the possible sets of initial conditions through multiple simulations; however, to verify the system unstable, existence of only one ever growing trajectory that remains in one cone at "all times" is sufficient. Fig. 5 demonstrates the stable case, where the trajectories are converging to the origin. Fig. 6 and Fig. 7 illustrates the unstable and oscillatory behavior, respectively. For the sake of presentation, we provide figures for only one initial condition in the case of stable and oscillatory designs. The phenomenon observed here, that all negative eigenvalues (in all four modes) may result in a conditionally stable or unstable systems, is observed in 2D PLSs as well.

\section{Conclusion And Future Directions}

The characterization of transitive cones obtained in Theorem 1 easily extends to A-matrices with repeated eigenvalues but having a diagonal Jordan form. It is also possible to derive similar results for A-matrices having arbitrary Jordan forms and A-matrices with a conjugate pair of eigenvalues. 


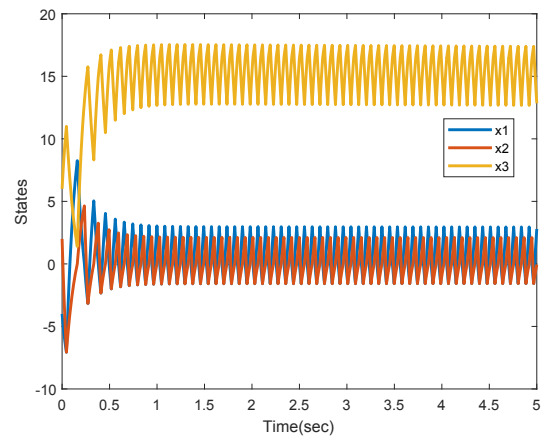

Fig. 7: State trajectories in oscillatory case

This paper focused only on the case of real distinct eigenvalues since it is presently difficult to bind all these sporadic results together in a concise treatment.

The assumption of mutually exclusive cones of boundary vectors and eigenvectors seem to open the way to extend Corollary 1 to arbitrary dimensional cones. Our future efforts will be directed towards such extensions.

\section{REFERENCES}

[1] A. Abdullahi, "Stability of planar piecewise linear systems: A geometric approach," Master's thesis, Bilkent University, 2015.

[2] M. K. Camlibel, W. Heemels, and J. Schumacher, "Algebraic necessary and sufficient conditions for the controllability of conewise linear systems," IEEE Transactions on Automatic Control, vol. 53, no. 3, pp. 762-774, 2008.

[3] M. K. Camlibel, J.-S. Pang, and J. Shen, "Conewise linear systems: non-zenoness and observability," SIAM Journal on Control and Optimization, vol. 45, no. 5, pp. 1769-1800, 2006.

[4] M. Camlibel, W. Heemels, and J. Schumacher, "Stability and controllability of planar bimodal linear complementarity systems," in Decision and Control, 2003. Proceedings. 42nd IEEE Conference on, vol. 2. IEEE, 2003, pp. 1651-1656.

[5] V. Eldem and I. Öner, "A note on the stability of bimodal systems in with discontinuous vector fields," International Journal of Control, vol. 88, no. 4, pp. 729-744, 2015.

[6] V. Eldem and G. Şahan, "Structure and stability of bimodal systems in R3: Part I," Appl. Comput. Math, vol. 13, no. 2, pp. 206-229, 2014.

[7] B. Grünbaum, V. Klee, M. A. Perles, and G. C. Shephard, Convex Polytopes. Springer, 1967.

[8] R. Iervolino, F. Vasca, and L. Iannelli, "Cone-copositive piecewise quadratic lyapunov functions for conewise linear systems," IEEE Transactions on Automatic Control, vol. 60, no. 11, pp. 3077-3082, 2015.

[9] J.-i. Imura and A. Van Der Schaft, "Characterization of well-posedness of piecewise-linear systems," IEEE Transactions on Automatic Control, vol. 45, no. 9, pp. 1600-1619, 2000.

[10] Y. Iwatani and S. Hara, "Stability tests and stabilization for piecewise linear systems based on poles and zeros of subsystems," Automatica, vol. 42, no. 10, pp. 1685-1695, 2006.

[11] M. K.-J. Johansson, Piecewise Linear Control Systems: A Computational Approach. Springer, 2003, vol. 284.

[12] D. Liberzon, "Switching in Systems and Control". Springer Science \& Business Media, 2003.

[13] D. Liberzon and A. S. Morse, "Basic problems in stability and design of switched systems," IEEE Control systems, vol. 19, no. 5, pp. 59-70, 1999.

[14] H. Lin and P. J. Antsaklis, "Stability and stabilizability of switched linear systems: a survey of recent results," IEEE Transactions on Automatic control, vol. 54, no. 2, pp. 308-322, 2009.

[15] Z. Sun, "Stability of piecewise linear systems revisited," Annual Reviews in Control, vol. 34, no. 2, pp. 221-231, 2010.

[16] Z. Sun and S. S. Ge, Stability Theory of Switched Dynamical Systems. Springer Science \& Business Media, 2011.

\section{Appendix: Proof of Lemma 1}

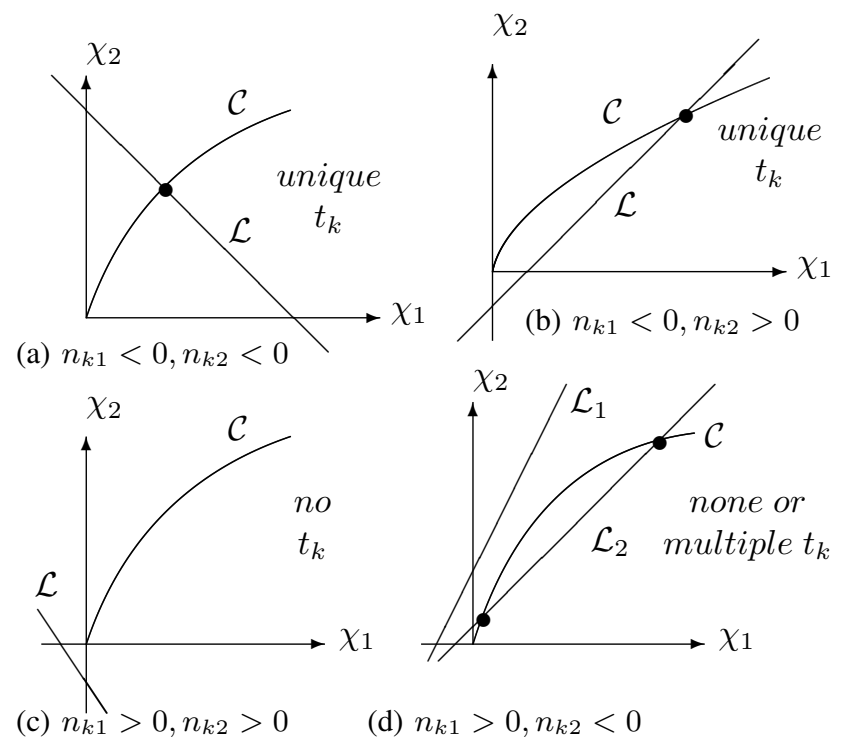

Fig. 8: Existence of $t_{k}$ when $\mathbf{c}_{k}^{T} \mathbf{b}>0$

Note that $n_{k i}=0$ only when $\mathbf{w}_{i}^{T} \mathbf{b}=0$, by (3), or equivalently, when $\mathbf{b}$ is on the $\mathbf{v}_{h} \mathbf{v}_{j}$-plane, with $(h, i, j)$ being a permutation of $(1,2,3)$. In such a case, the trajectory that starts with $\mathbf{b}$ remains in the $\mathbf{v}_{h} \mathbf{v}_{j}$-plane for all $t \geq 0$. Also since, by $\mathbf{v}_{1} \mathbf{w}_{1}^{T}+\mathbf{v}_{2} \mathbf{w}_{2}^{T}+\mathbf{v}_{3} \mathbf{w}_{3}^{T}=I$, we have $n_{k 1}+n_{k 2}+n_{k 3}=\mathbf{c}_{k}^{T} \mathbf{b}$, the equality $\mathbf{c}_{k}^{T} \mathbf{x}(t, \mathbf{b})=0$ holds if and only if

$$
\mathcal{L}: \chi_{1} n_{k 1}+\chi_{2} n_{k 2}+\mathbf{c}_{k}^{T} \mathbf{b}=0,
$$

where

$$
\chi_{1}:=e^{\left(\lambda_{1}-\lambda_{3}\right) t}-1 \geq 0, \chi_{2}:=e^{\left(\lambda_{2}-\lambda_{3}\right) t}-1 \geq 0, \forall t \geq 0 .
$$

These time-dependent variables are related by

$$
\mathcal{C}: \chi_{2}=\left(\chi_{1}+1\right)^{p}-1,
$$

which describes a parametric curve that monotonically increases with curvature downwards in $\chi_{1} \chi_{2}$-plane as shown in Fig. 8. Thus, an intersection with $\mathbf{s}_{l} \mathbf{s}_{m}$-plane, i.e., the border $\mathrm{B}_{k}$, at a finite time occurs if and only if the line $\mathcal{L}$ of (7) intersects the curve $\mathcal{C}$ of (8) in the first quadrant of the $\chi_{1} \chi_{2}$-plane. The value of the parameter $t>0$ at such an intersection is set equal to $t_{k}$. If, in addition, the two inequalities $\mathbf{c}_{l}^{T} \mathbf{x}\left(t_{k}, \mathbf{b}\right)=>0, \mathbf{c}_{m}^{T} \mathbf{x}\left(t_{k}, \mathbf{b}\right)=>0$, also hold for all $t \in\left(0, t_{k}\right)$, then $t_{k}$ is the first time instant at which the trajectory intersects a border (among the three) so that $t_{k}=t_{k}^{*}$ in the context of Definition 2(iii).

Fig. 8 examines whether $\mathcal{L}$ and $\mathcal{C}$ intersect under four possible sign patterns for $n_{k 1}$ and $n_{k 2}$. If $n_{k 1}<0$, then they intersect for both positive and negative $n_{k 2}$, as illustrated in Figures $8(\mathrm{a})$ and $8(\mathrm{~b})$. If $n_{k 1}>0$, then no intersection exists when $n_{k 2}>0$ and the intersection conditionally exists when $n_{k 2}<0$. These cases are shown in Figures 8(c) and (d). 